\title{
Rising Trends of Multidrug Resistant (MDR) Tuberculosis In Pakistan
}

\author{
Ahsan Sattar Sheikh*1, Mubashar Aziz² and Mazhar Ayaz ${ }^{2}$ \\ ${ }^{1}$ Institute of Food Science and Nutrition, Pakistan \\ ${ }^{2}$ Department of Pathobiology, Faculty of Veterinary Sciences, Pakistan
}

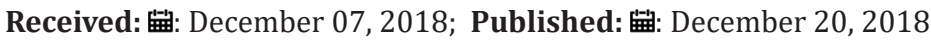

*Corresponding author: Ahsan Sattar Sheikh, Institute of Food Science and Nutrition, Pakistan

\begin{abstract}
Worldwide more than 10.4 million persons are infected with Mycobacterium species - the causative agent each year. Of these all, 1.7 million patients loss their life each year. Patients with tuberculosis (TB) come from low and middle income countries. Today this active surge in TB cases comes from 22 high burden countries (HBC). To date, Mycobacterium species whether they are conventional or non-conventional TB, its spread within worldwide geographic regions / countries doesn't show any traceable genetic mechanism of evolution. With this world health authorities projects emergence of more than 600 thousand new antibiotic resistant cases to first line primary drugs in Anti Tuberculosis Therapy (ATT) in HBC. It is calculated that from all world rifampicin resistant TB, $47 \%$ of these patients live India, Pakistan, China and Russian Federation. In the first Pakistan National Anti-Tuberculosis Drug Resistance survey reports (2012-2013) indicates that Pakistan has 3.6\% MDR - TB cases. Pakistan is included in seven countries that shares more than $2 / 3$ of all diagnosed cases. In the high burden countries several socio-economic and biological factors including poverty, place of residence, place of consultation, level of education, wait time for available health care facility contribute to emerging trends in chemotherapy appliance.

In the Sustainable Development Goals (SDGs) devised by world agencies drills to all nations to End TB by 2035. Recently, PCR based GenoType MTBD plus assay for MDR cases show mutations at position G944C with S315T amino acid changes. In recent years development of miRNA against several transcriptional factors have been identified. Some other microRNAs include miR-204 that have shown to effect on signalling pathways for pulmonary arterial smooth muscle cells activities
\end{abstract}

Abbreviations: TB: Tuberculosis; ATT: Anti Tuberculosis Therapy; HBC: High Burden Countries; SDGs: Sustainable Development Goals; MIRUVNTR: Interspersed Repetitive Units - Variable Number of Tandem Repeats; MDR-TB: Multi Drug Resistant -TB; BAL: Broncho alveolar lavage; GA: Gastric Aspiration; TBIC: TB Infection Control

\section{Introduction}

Documented Tuberculosis (TB) history epochs back to the prehistoric Egypt. Several paintings and preserved mummies tell us that TB was around in the era. Hippocrates, the scholar, used the phases "pthisis" for Tuberculosis that means "to dry up". In Chinese literature also TB is well mentioned in the $2700 \mathrm{BC}$ era of Emperor Shenong. During various regimes, such as in Rigveda era (1500 BC) it was called "yaksma" that was followed by term "balasa" and "scrofula" in the Indian literature. In 17th century European call it "great white plaque". The word Tuberculosis was proposed by one German Professor in his book "Systematik de speziellen Pathologie und Therapie" for the first time. Tuberculosis is top ten causes of worldwide mortality. Globally, more than 10.4 million persons were infected with Mycobacterium species - the causative agent each year. Of all these, 1.7 million losses their life. Almost 95\% of these people live in low and middle income countries [1]. Additional to above, nearly 1 million children also contribute to the TB burden $[2,3]$. Estimates suggest that worldwide tuberculosis mortality by $3 \%$ and incidence by $2 \%$ annually. The main surge of TB cases comes from 22 high burden countries [4]. WHO has created targets this high burden upsurge to end by 2030. It is suggestive to coupe with this epidemic by taking measures to reduce tuberculosis by 4-5\% to equate the rising recent trends [5]. Since then, more global funds have been allocated which are now more than doubled to US\$ 6.9 billion in 2017 [6,7]. 
In developing countries large number of infectious diseases affects general masses [6]. From these tuberculosis is identified as one of the disease that is affecting especially low prominence people [8]. To date, Mycobacterium species, conventional and nonconventional, spread within many geographic regions / countries of the world with no traceable genetic mechanism of evolution. Recent work of Ali and coworkers at Agha Khan Hospital, Karachi, Pakistan rightly indicated that Interspersed Repetitive Units Variable Number of Tandem Repeats (MIRU-VNTR) typing can be used discriminate various 24 loci to map evolution [9]. World health bodies are projecting more than 600 thousand new resistant cases emergence to first line primary drugs used in Anti Tuberculosis Therapy (ATT) in high burden countries. If this happens, it will create devastation to these nations $[10,11]$. Pakistan shares a very high percentage of Multi Drug Resistance (MDR)-TB in the WHO-Mediterranean region $[10,12,13]$. It is calculated that from all world rifampicin resistant TB, $47 \%$ of these patients live India, Pakistan, China and Russian Federation. Furthermore, community (Pakistan) based notes share the presence of resistant / resilient TB cases could foresee an unpredictable, incomplete statistics, path for sustained treatment $[13,14]$. In the 1st Pakistan National Anti-Tuberculosis Drug Resistance survey reports (2012-2013) indicates that Pakistan has 3.6\% MDR - TB case patients [13]. From these $18.1 \%$ were placed in treated class patients [15]. With these figures, Pakistan is included in seven countries that shared more than $2 / 3$ all diagnosed cases in 2016 [11,16].

From various studies, it is ascertained to some conviction that young people are at higher risk to contract the disease. This is due to poor food habits / nutrients that reduces immunity levels in the age group, especially in $10-25$ years $[17,11]$. Province wise, highest numbers of MDR cases were reported in Punjab (51\%) which is followed by Sindh (23\%), 15\% in Khyber Pakhtunkhwa and 3.5\% in Baluchistan [18]. Analysis on the increasing MDR - TB cases suggest that most of the time they are linked to treatment failure, relapse, complications and death [10]. These figures / trends on MDR have also shaken the foundations of National TB control programs [18]. In Pakistan, Statistics on Multi Drug Resistant - TB (MDR-TB) also produced shock waves to WHO which created a health crisis and security hazard issue in the region [WHO, 2017]. Today, Pakistan is ranked 5th in TB burden country with 6th in largest MDR - TB cases [20]. It is included in 27 countries that have high MDR burden countries [11]. Key reasons for emergence of such drug resistance form include: delays in diagnosis, unsupervised, inappropriate and inadequate drug regimens, poor follow-up and lack of a social support programme for high-risk populations [4]. Analysis and comparison of old and new data suggest that a relatively high percentage of rifampicin drug resistance $(4.2 \%)$ in new TB cases, followed by almost four times in previously treated cases.

This is itself a bombshell in the making. On top of it, XDR (extensively Drug Resistance)-TB is also seen as much higher percentage that is a peril for community in future [10]. XDR is an advanced form of MDR-TB that also involves fluoroquinolones and resistance to at least one out of three injectable second line ATT i.e.
Amikacin, Kanamycin or Capreomycin [13,21]. Information from Karachi tell us that MDR-TB isolates share 27\% fluoroquinolone resistance [13]. Another important comparative study showed that only $17.4 \%$ MDR cases were reported in 2005 as against $53.9 \%$ in 2015 (three times increase) [19,22]. Interestingly, Shakoor and coworkers, however, showed that presence of this fluoroquinolone resistance is not related to the volume of its consumption [23]. Addition to this misery, absence of insufficient disease burden, accurate detailed data on TB morbidity, its incidence, prevalence and mortality are all missing for referral $[18,24,25]$. Development of multidimensional antibiotic resistance spread created an extensive overlay to be cared worldwide. This has shaped pharmaceutical research on war footing to develop new antibiotics for clinical use. Looking at the situation, WHO also prioritized several programs. This includes selection of 44 newly developed intravenous antibiotics. From these, only 15 show some potential.

Today just three got green signal to go ahead out of 15 to move to phase 3 trial stages. Only two groups i.e. Lipopeptides and Oxazolidinones, are now been approved by American Drug Administration and European Medicines Agency [26]. Sustainable Development Goals (SDGs) and Tuberculosis: The Sustainable Development Goals (SDGs) devised by world body instructs all nations to End TB by 2035 [27]. In 2016, most tuberculosis cases occurred in WHO South East Asia region (45\%), 25\% in WHO African region, $17 \%$ in WHO Western Pacific region and small proportion in WHO Eastern Mediterranean region [WHO report 2017]. The pulmonary TB, specially, showed an evolutionary off shoots in tree, especially, with MDR-TB and XDR. MDR - TB or XDR - TB are defined as a type of infection by Mycobacterium that show resistance to at least two first line anti-TB drugs i.e. isoniazid and rifampicin or at least resistance to four first line drugs [11]. Recently, PCR based Geno Type MTBD plus assay for MDR cases show mutation at position G944C with Ser315Thr amino acid change [28]. Globally, these MDR - TB cases are continuously reported both in industrialized and developing nations.

In the high burden countries several socio-economic and biological factors including poverty, place of residence, place of consultation, level of education, waiting time for available health care facility contribute to emerging trends in chemotherapy appliance [14], low vigilance, and smoking habits are to blamed for high TB prevalence [10,29]. Most of the early diagnosis of TB in developing countries, like in Pakistan, is very much dependent on the conventional microscopic methods [14]. It is suggestive that number of specimens other than sputum could result in much faster and precise diagnosis. Because of high illiteracy, good quality sputum, sputum induction is nearly an impossible task in hospitals and clinics. Specimen collected by Broncho alveolar lavage (BAL) or gastric aspiration (GA) provides more conclusive results then routine sputum microscopy. Both BAL and GA even some time provide better results than TB culture [20]. To improve the diagnosis, Gene Xpert ${ }^{\circledR}$ (Cepheid, USA) was inducted into the health care system of Pakistan. The PCR methodology is used both in detection of Mycobacterium tuberculosis and mutations 
in rifampin resistance since 2011 [1]. Since 2015, this molecular technique is now upgraded to 60 centers with the financial help of WHO. These centers are providing excellent data, especially for rifampin MDR -TB cases [30].

Very importantly, spread of tuberculosis within the healthcare settings is also alarming.

TB Infection Control (TBIC) measures / programs that were implemented by WHO infection control are extremely important to completely eradicate TB and MDR / XDR - TB within health facilities [31]. Lack of specific microbiological culturing facilities (BSL III Laboratory) make it very difficult, especially country like Pakistan, to not only proves presence of Mycobacterium MDR - TB in patents $[14,30]$. However, number of studies is conducted in Pakistan to assess the MDR or even XDR cases. One of our study on MDR / XDR case in Faisalabad, we demonstrated that Mycobacterium species isolated from clinically difficult to treat patients were completely resistant to Isoniazid, and $84 \%$ of isolates were rifampin resistant [32]. Several techniques are in use to combat the menace of tuberculosis in recent years. Development of miRNA against several transcriptional factors have been identified with databases viz, miRDB, miRNAMap, miRanda-mirSVR, and DIANAmicroT [33]. The microRNAs, a new group in RNA class, have emerged as important regulator of pre- and posttranscriptional gene modulator / regulator [34]. Scientists are taking new directions to consider the importance of many miRNAs in pulmonary hypertensive patients. Like miR-150, upregulation is an independent predictor an adverse outcome. Some other microRNAs include miR-204 that have shown to effect on signalling pathways for pulmonary arterial smooth muscle cells activities [33]. National Tuberculosis program of Pakistan should also adopt measures not only conservative methods of $20^{\text {th }}$ century but also in addition add more $21^{\text {st }}$ century technology to jointly remove this disease from the face of earth.

\section{References}

1. Irfan Ullah, Javaid A, Masud H, Ali M, Basit A, et al. (2017) Rapid detection of Mycobacterium tuberculosis and rifampicin resistance in extrapulmonary tuberculosis and sputum smear-negative pulmonary suspects using Xpert MTB/RI. Journal of Medical Microbiology 66(4) :412-418.

2. Ahluwalia PK, Pandey RK, Sehajpal PK, Prajapati VK (2017) Perturbed microRNA Expression by Mycobacterium tuberculosis Promotes Macrophage Polarization Leading to Pro-survival Foam Cell. Front. Immunol 8: 107.

3. Floyd K, Glaziou P, Houben RMGJ, Sumner T, White RG, et al. (2018) Global tuberculosis targets and milestones set for 2016-2035: definition and rationale. Int J Tuberc Lung Dis 22(7): 723-730.

4. WHO, Tuberculosis. World Health Organization Regional office for the Eastern Mediterranean.

5. Khademi Farzad, Arshid Yousefi Avarvand, Mohammad Derakhshan, Hamid Vaez, Ramin Sadeghi3 (2017) Middle East Mycobacterium tuberculosis Antibiotic Resistance: A Systematic Review and MetaAnalysis Infect Epidemiol Med 3(1): 25-35.

6. Jones KE, Patel NG, Levy MA, Storeygard A, Balk D, et al. (2008) Global trends in emerging infectious diseases. Nature. volume 451: 990-993.

7. Chauhan V, Thakur S (2017) State of the Globe: The Global Battle for Survival against Mycobacterium tuberculosis. J Glob Infect Dis 9(4): 129130.
8. Monge Maillo B, Lopaz Velez R (2017) Challenges in the management of Chagas disease in Latin-American migrants in Europe. Clin Microbiol Infec 23(5): 290-295.

9. Ali A, Hasan Z, Jafri S, Inayat R, Hasan R (2014) Mycobacterium tuberculosis Central Asian Strain (CAS) lineage strains in Pakistan reveal lower diversity of MIRU loci than other strains. Intl J Mycobacteriol 3(2): 108-116.

10. Ahmad N, Javaid A, Azhar S, Sulaiman S, Mingc LC, et al. (2016) Resistance patterns, prevalence, and predictors of fluoroquinolones resistance in multidrug resistant tuberculosis patients. Brazilian J Infect Dis 20(1): 41-47.

11. Khurram M, Khaar HTB, Fahim M (2012) Multidrug-resistant tuberculosis in Rawalpindi, Pakistan. J Infect Dev Ctries 6(1): 29-32.

12. Pakistan WHO (2014) Antimicrobial resistance: global report on surveillance 2014. Geneva: World Health Organization.

13. Zaidi SMA, Haseeb A, Habib SS, Malik A, Khowaja S, et al. (2017) Emergence of fluoroquinolone resistance among drug resistant tuberculosis patients at a tertiary care facility in Karachi BMC Res Notes 10(1): 313.

14. Khan AH (2017) Tuberculosis control in Sindh, Pakistan: Critical analysis of its implementation. Journal of Infection and Public Health 10(1): 1-7.

15. Qadeer E (2017) Tuberculosis Control in Pakistan - Module for MBBS Students published by National Tuberculosis Program, Pakistan.

16. Javaid A, Ahmad N, Khan AH, Shaheen Z (2017) Applicability of the World Health Organization recommended new shorter regimen in a multidrugresistant tuberculosis high burden country. Eur Respir J 49: 1601967.

17. Irfan Ullah, Javaid A, Tahir Z, Obaid Ullah, Shah AA, et al. (2016). Pattern of Drug Resistance and Risk Factors Associated with Development of Drug Resistant Mycobacterium tuberculosis in Pakistan. Plos ONE.

18. Khan WM, Smith H, Qadeer E, Hassounah S (2016) Knowledge and perceptions of national and provincial tuberculosis control programme managers in Pakistan about the WHO Stop TB strategy: a qualitative study. Journal of the Royal Society of Medicine 8(1) 1-9.

19. Ahmad N, Khan AH, Sulaiman SA, Javaid A (2015) Fluoroquinolones resistance in multidrug-resistant tuberculosis in Pakistan and suitability of guidelines recommended standardized regimen. Int J Mycobacteriol 4(3): 258-259.

20. Aslam W, Tahseen S, Schomotzer C, Hussain A, Khanzada F, et al. (2017) Gastric specimens for diagnosing tuberculosis in adults unable to expectorate in Rawalpindi, Pakistan Public Health Action 7: 141-146.

21. Munir Muhammad Kashif, Rehman Sana, Iqbal Rizwan (2018) Meeting the Challenge, Making a Difference: Multidrug Resistance Tuberculosis in Pakistan Pak J Med Res 57(1): 1-2.

22. Sotgiu G, Migliori GB (2017) Group 5 drugs for multidrug-resistant tuberculosis: is the glass half full or half empty? Eur Respir J 49: 1602273.

23. Shakoor S, Tahseen S, Jabeen K, Fatima R, Malik FR, et al. (2016) Fluoroquinolone consumption and esistance trends in Mycobacterium tuberculosis and other respiratory pathogens: Ecological antibiotic pressure and consequences in Pakistan, 2009-2015. Intl J Mycobacteriol 5(4): 412-416.

24. Qasim M (2016) TB kills over 70,000 people every year in Pakistan. The News 24 march, 2016 Islamadad, Pakistan.

25. Hussain A, Mirza Z, Qureshi FA, Hafeez A (2005) Adherence of private practitioners with the National Tuberculosis treatment should build close collaboration with international guidelines in Pakistan: a survey report. J Pak Med Assoc 55(1): 17-19.

26. Tacconelli E, Mendelson M, Cammeli Y, Outterson K, Monnet DL, et al. (2017) Discovery, research, and development of new antibiotics: the WHO prioiity list of antibiotic-resistant bacteria and tuberculosis. Lancet (Infectious Diseases 18(3): 318-327. 
27. Olea Popelka F, Muwonge A, Perera A, Dean AS, Mumford E, et al. (2017) Tip of the iceberg: Zoonotic tuberculosis in humans caused by Mycobacterium bovis - a call for action. The Lancet Infectious Diseases 17(1): 21-25.

28. Javed H, Jamil N, Jagielski T, Bakułab Z (2016) Evaluation of genotype MTBDRplus assay for rapid detection of isoniazid and rifampicin resistance in Mycobacterium tuberculosis clinical isolates from Pakistan. Int e rnat i o na l J. Mycobacteriol 5(1): S147 -S148.

29. Hashmi HJ, Javed H, Jamil N (2017) Emerging epidemic of drug resistant tuberculosis in vulnerable population of developing countries. Afri Health Sci 17(2): 599-602.

30. Ahmad N, Khan AH, Sulaiman SA, Javaid A (2015) Fluoroquinolones resistance in multidrug-resistant tuberculosis in Pakistan and suitability of guidelines recommended standardized regimen. Int J Mycobacteriol 4(3): 258-259.

31. Waheed MA Khan, R Fatima, A Yaqoob, A Mirza, E Qadeer, et al. (2017) Infection control in hospitals managing drug-resistant tuberculosis in Pakistan: how are we doing? Public Health Action 7(1): 27-31.

32. Sheikh AS, Jafri SA, Asif M, Ahmad S (2004) Isolation of Mycobacterium species on synthetic media, and sensitivity of 7 anti-tuberculosis drugs in Faisalabad Region. Pak J Biochem Mol Biol 37: 35-40.

33. Yanga T, Ge B (2018) miRNAs in immune responses to Mycobacterium tuberculosis infection. Cancer Letters 431: 22-30.

34. Kim JK, Kim TS, Basu J, Jo EK (2017) MicroRNA in innate immunity and autophagy during mycobacterial infection. Cellular Microbiology 19(1): e12687.

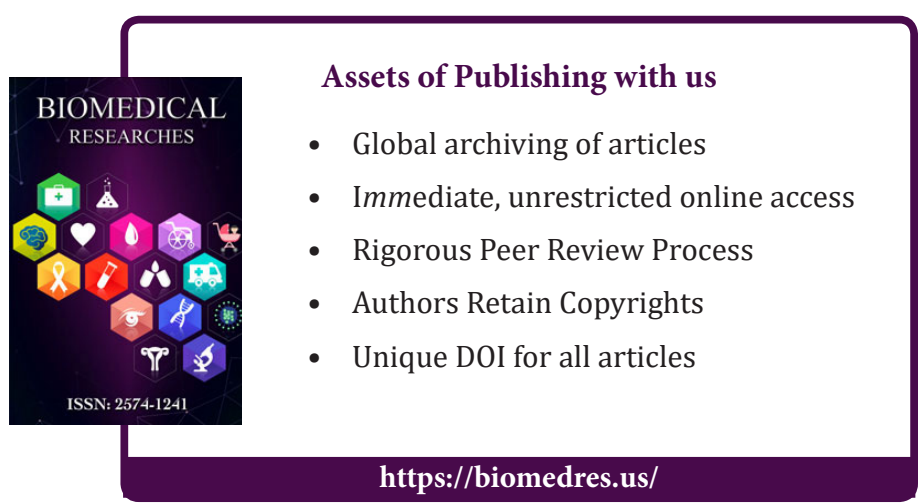

ISSN: 2574-1241

DOI: $10.26717 / B J S T R .2018 .12 .002254$

Ahsan Sattar Sheikh. Biomed J Sci \& Tech Res

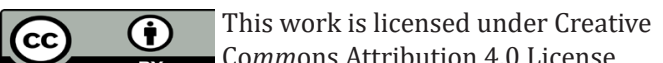

Submission Link: https://biomedres.us/submit-manuscript.php 